\title{
SOME LANDAU TYPE INEQUALITIES FOR FUNCTIONS \\ WHOSE DERIVATIVES ARE OF LOCALLY BOUNDED VARIATION
}

\author{
N. S. BARNETT AND S. S. DRAGOMIR
}

Abstract. Some inequalities of the Landau type for functions whose derivatives are of locally bounded variation are pointed out.

\section{Introduction}

The following version of Ostrowski's inequality for functions of bounded variation was obtained by the second author in [2] (see also [3]):

Theorem 1. Let $\varphi:[a, b] \rightarrow \mathbb{R}$ be a function of bounded variation on $[a, b]$. Then for any $x \in[a, b]$ one has the inequality:

$$
\left|\varphi(x)-\frac{1}{b-a} \int_{a}^{b} \varphi(t) d t\right| \leq\left[\frac{1}{2}+\frac{\left|x-\frac{a+b}{2}\right|}{b-a}\right] \bigvee_{a}^{b}(\varphi),
$$

where $\bigvee_{a}^{b}(\varphi)$ denotes the total variation of $\varphi$ on $[a, b]$. The constant $\frac{1}{2}$ is the best possible.

We now recall some classical results due to Landau [8].

Let $I=\mathbb{R}_{+}$or $I=\mathbb{R}$. If $f: I \rightarrow \mathbb{R}$ is twice differentiable and $f, f^{\prime \prime} \in L_{p}(I)$, $p \in[1, \infty]$, then $f^{\prime} \in L_{p}(I)$. Moreover, there exists a constant $C_{p}(I)>0$ independent of the function $f$, such that

$$
\left\|f^{\prime}\right\|_{I, p} \leq C_{p}(I)\|f\|_{I, p}^{\frac{1}{2}}\left\|f^{\prime \prime}\right\|_{I, p}^{\frac{1}{2}},
$$

where $\|\cdot\|_{I, p}$ is the $p$-norm on the interval $I$, i.e., we recall

$$
\|h\|_{I, \infty}:=e s s \sup _{t \in I}|h(t)|,
$$

and

$$
\|h\|_{I, p}:=\left(\int_{I}|h(t)|^{p} d t\right)^{\frac{1}{p}} \quad \text { if } \quad p \in[1, \infty) .
$$

Received February 15, 2005.

2000 Mathematics Subject Classification. Primary 26D15; Secondary 26D10.

Key words and phrases. Landau inequality, functions of bounded variation. 
Landau considered the case $p=\infty$ and proved that

$$
C_{\infty}\left(\mathbb{R}_{+}\right)=2 \text { and } C_{\infty}(\mathbb{R})=\sqrt{2}
$$

are the best constants for which (1.2) holds.

In 1932, G.H. Hardy and J. E. Littlewood [5] proved (1.2) for $p=2$, with the best constants

$$
C_{2}\left(\mathbb{R}_{+}\right)=\sqrt{2} \text { and } C_{2}(\mathbb{R})=1 .
$$

In 1935, G. H. Hardy, E. Landau and J. E. Littlewood [6] showed that the best constant $C_{p}\left(\mathbb{R}_{+}\right)$in (1.2) satisfies the estimate

$$
C_{p}\left(\mathbb{R}_{+}\right) \leq 2 \text { for } p \in[1, \infty),
$$

which yields $C_{p}(\mathbb{R}) \leq 2$ for $p \in[1, \infty)$. Actually, as shown in [7] and [1], $C_{p}(\mathbb{R}) \leq \sqrt{2}$.

In this paper, by the use of the inequality (1.1), we point out some Landau type results for arbitrary subintervals $I$ of $\mathbb{R}$ and under more relaxed assumptions on the derivative $f^{\prime}$.

\section{A Technical Lemma}

The following technical lemma, that is important in the sequel, holds [4]. For the sake of completeness, a short proof is outlined below.

Lemma 1. Let $C, D>0$ and $r, u \in(0,1]$. Consider the function $g_{r, u}:(0, \infty] \rightarrow \mathbb{R}$ given by

$$
g_{r, u}(\lambda)=\frac{C}{\lambda^{u}}+D \lambda^{r} .
$$

Define

$$
\lambda_{0}:=\left(\frac{u C}{r D}\right)^{\frac{1}{r+u}} \in(0, \infty),
$$

then for $\lambda_{1} \in(0, \infty)$ we have,

$$
\inf _{\lambda \in\left(0, \lambda_{1}\right]} g_{r, u}(\lambda)= \begin{cases}\frac{r+u}{u^{\frac{u}{r+u}} \cdot r^{\frac{u}{r+u}}} C^{\frac{r}{r+u}} D^{\frac{r}{r+u}} & \text { if } \lambda_{1} \geq \lambda_{0}, \\ \frac{C}{\lambda_{1}^{u}}+D \lambda_{1}^{r} & \text { if } 0<\lambda_{1}<\lambda_{0} .\end{cases}
$$

Proof. We observe that

$$
g_{r, u}^{\prime}(\lambda)=\frac{r D \lambda^{r+u}-C u}{\lambda^{u+1}}, \quad \lambda \in(0, \infty) .
$$

The unique solution of the equation $g_{r, u}^{\prime}(\lambda)=0, \lambda \in(0, \infty)$ is

$$
\lambda_{0}=\left(\frac{u C}{r D}\right)^{\frac{1}{r+u}} \in(0, \infty) .
$$


The function $g_{r, u}$ is decreasing on $\left(0, \lambda_{0}\right)$ and increasing on $\left(\lambda_{0}, \infty\right)$. The global minimum for $g_{r, u}$ on $(0, \infty)$ is

$$
\begin{aligned}
g_{r, u}\left(\lambda_{0}\right) & =\frac{C}{\left(\frac{u C}{r D}\right)^{\frac{u}{r+u}}}+D \cdot\left(\frac{u C}{r D}\right)^{\frac{r}{r+u}} \\
& =\frac{r+u}{u^{\frac{u}{r+u}} r^{\frac{r}{r+u}}} \cdot C^{\frac{r}{r+u}} D^{\frac{u}{r+u}}
\end{aligned}
$$

which proves $(2.2)$.

The following particular cases are useful.

Corollary 1. Let $C, D>0$ and $r \in(0,1]$. Consider the function $g_{r}:(0, \infty) \rightarrow \mathbb{R}$, given by

$$
g_{r}(\lambda)=\frac{C}{\lambda}+D \lambda^{r}
$$

Define

$$
\overline{\lambda_{0}}=\left(\frac{C}{r D}\right)^{\frac{1}{r+1}} \in(0, \infty)
$$

then for $\lambda_{1} \in(0, \infty)$,

$$
\inf _{\lambda \in\left(0, \lambda_{1}\right]} g_{r}(\lambda)= \begin{cases}\frac{r+1}{r^{r+u}} C^{\frac{r}{r+1}} D^{\frac{1}{r+1}} & \text { if } \lambda_{1} \geq \overline{\lambda_{0}}, \\ \frac{C}{\lambda_{1}}+D \lambda_{1}^{r} & \text { if } 0<\lambda_{1}<\overline{\lambda_{0}} .\end{cases}
$$

Corollary 2. Let $C, D>0$ and $u \in(0,1]$. Consider the function $g_{u}:(0, \infty) \rightarrow \mathbb{R}$ given by

$$
g_{u}(\lambda)=\frac{C}{\lambda^{u}}+D \lambda
$$

Define

$$
\widetilde{\lambda_{0}}=\left(\frac{u C}{D}\right)^{\frac{1}{1+u}} \in(0, \infty)
$$

then for $\lambda_{1} \in(0, \infty)$,

$$
\inf _{\lambda \in\left(0, \lambda_{1}\right]} g_{u}(\lambda)= \begin{cases}\frac{1+u}{u^{\frac{u}{1+u}}} C^{\frac{1}{u+1}} D^{\frac{u}{u+1}} & \text { if } \lambda_{1} \geq \widetilde{\lambda_{0}} \\ \frac{C}{\lambda_{1}^{u}}+D \lambda_{1} & \text { if } 0<\lambda_{1}<\widetilde{\lambda_{0}}\end{cases}
$$

Remark 1. If $r=u=1$, then the following result holds:

$$
\inf _{\lambda \in\left(0, \lambda_{1}\right]}\left(\frac{C}{\lambda}+D \lambda\right)=\left\{\begin{array}{l}
2 \sqrt{C D} \quad \text { if } \lambda_{1} \geq \sqrt{\frac{C}{D}} \\
\frac{C}{\lambda_{1}}+D \lambda_{1} \text { if } 0<\lambda_{1}<\sqrt{\frac{C}{D}} .
\end{array}\right.
$$


3. The Case When $f \in L_{\infty}(I)$

The following theorem holds.

Theorem 2. Let $I$ be an interval in $\mathbb{R}$ and $f: I \rightarrow \mathbb{R}$ a locally absolutely continuous function on I. If $f \in L_{\infty}(I)$, the derivative $f^{\prime}: I \rightarrow \mathbb{R}$ is of locally bounded variation and there exists a constant $V_{I}>0$ and $r \in(0,1]$ such that

$$
\left|\bigvee_{a}^{b}\left(g^{\prime}\right)\right| \leq V_{I}|a-b|^{r} \quad \text { for any } a, b \in I
$$

then $f^{\prime} \in L_{\infty}(I)$ and

$$
\left\|f^{\prime}\right\|_{I, \infty} \leq\left\{\begin{array}{l}
\frac{2^{\frac{r}{r+1}}(r+1)}{r^{\frac{r}{r+1}}}\|f\|_{I, \infty}^{\frac{r}{r+1}} V_{I}^{\frac{1}{r+1}} \text { if } m(I) \geq \frac{2^{\frac{r+2}{r+1}}\|f\|_{I, \infty}^{\frac{r}{r+1}}}{r^{\frac{1}{r+1}} V_{I}^{\frac{1}{r+1}}} \\
\frac{4\|f\|_{I, \infty}}{m(I)}+\frac{V_{I}(m(I))^{r}}{2^{r}} \quad \text { if } 0<m(I)<\frac{2^{\frac{r+2}{r+1}}\|f\|_{I, \infty}^{\frac{r}{r+1}}}{r^{\frac{1}{r+1}} V_{I}^{\frac{1}{r+1}}}
\end{array}\right.
$$

Proof. Applying (1.1) for $\varphi=f^{\prime}$, we deduce

$$
\left|f^{\prime}(x)\right| \leq\left|\frac{f(b)-f(a)}{b-a}\right|+\left[\frac{1}{2}+\frac{\left|x-\frac{a+b}{2}\right|}{b-a}\right]\left|\bigvee_{a}^{b}\left(f^{\prime}\right)\right|
$$

for any $a, b \in I, a \neq b$ and $x$ between them, giving, for $x=a$,

$$
\left|f^{\prime}(a)\right| \leq \frac{|f(b)-f(a)|}{|b-a|}+\left|\bigvee_{a}^{b}\left(f^{\prime}\right)\right|
$$

for any $a, b \in I, a \neq b$.

Using the hypothesis (3.1) and the fact that $f \in L_{\infty}(I)$, we conclude that

$$
\begin{aligned}
\left|f^{\prime}(a)\right| & \leq \frac{|f(b)-f(a)|}{|b-a|}+V_{I}|b-a|^{r} \\
& =\frac{2\|f\|_{I, \infty}}{|b-a|}+V_{I}|b-a|^{r}
\end{aligned}
$$

for almost every $a, b \in I, a \neq b$.

Now, observe that for any $a \in I$ and any $s \in\left(0, \frac{m(I)}{2}\right)$, there exists $b \in I$ such that $s=|b-a|$ and then, by (3.4),

$$
\left|f^{\prime}(a)\right| \leq \frac{2\|f\|_{I, \infty}}{s}+V_{I} s^{r}
$$


for almost any $a \in I$ and every $s \in\left(0, \frac{m(I)}{2}\right)$. By taking the infimum over $s$ on $\left(0, \frac{m(I)}{2}\right)$, we have,

$$
\left|f^{\prime}(a)\right| \leq \inf _{s \in\left(0, \frac{m(I)}{2}\right)}\left[\frac{2\|f\|_{I, \infty}}{s}+V_{I} s^{r}\right]=K
$$

for almost any $a \in I$.

If we take the essential supremum over $a \in I$ in (3.6), we conclude that

$$
\left\|f^{\prime}\right\|_{I, \infty} \leq K .
$$

Making use of Corollary 1, we get

$$
\begin{aligned}
K=\left\{\begin{array}{l}
\frac{r+1}{r^{\frac{r}{r+1}}}\left(2\|f\|_{I, \infty}\right)^{\frac{r}{r+1}} \cdot V_{I}^{\frac{1}{r+1}} \text { if } \frac{m(I)}{2} \geq\left(\frac{2\|f\|_{I, \infty}}{r V_{I}}\right)^{\frac{1}{r+1}} \\
\frac{2\|f\|_{I, \infty}}{\frac{m(I)}{2}}+V_{I}\left(\frac{m(I)}{2}\right)^{r} \text { if } \frac{m(I)}{2}<\left(\frac{2\|f\|_{I, \infty}}{r V_{I}}\right)^{\frac{1}{r+1}}
\end{array}\right. \\
= \begin{cases}\frac{2^{\frac{r}{r+1}}(r+1)}{r^{\frac{r}{r+1}}}\|f\|_{I, \infty}^{\frac{r}{r+1}} V_{I}^{\frac{1}{r+1}} \text { if } m(I) \geq \frac{2^{\frac{r+2}{r+1}}\|f\|_{I, \infty}^{\frac{r}{r+1}}}{r^{\frac{1}{r+1}} V_{I}^{\frac{1}{r+1}}} \\
\frac{4\|f\|_{I, \infty}}{m(I)}+\frac{V_{I}(m(I))^{r}}{2^{r}} & \text { if } 0<m(I)<\frac{2^{\frac{r+2}{r+1}}\|f\|_{I, \infty}^{\frac{r}{r+1}}}{r^{\frac{1}{r+1}} V_{I}^{\frac{1}{r+1}}}\end{cases}
\end{aligned}
$$

and the inequality (3.2) is obtained.

\section{The Case when $f$ is Hölder Continuous}

The following theorem holds.

Theorem 3. Let $I$ be an interval in $\mathbb{R}$ and $f: I \rightarrow \mathbb{R}$ a locally absolutely continuous function on $I$. If $f$ satisfies the Hölder condition

$$
|f(b)-f(a)| \leq K|b-a|^{\ell} \text { for any } a, b \in I,
$$

where $K>0$ and $\ell \in(0,1)$ are given, and the derivative $f^{\prime}: I \rightarrow \mathbb{R}$ is of locally bounded variation and the condition (3.1) holds, then $f^{\prime}$ is bounded in $I$ and

$$
\left\|f^{\prime}\right\|_{I, \infty} \leq \begin{cases}\frac{r+1-\ell}{(1-\ell)^{\frac{1-\ell}{r+1-\ell}} r^{\frac{r}{r+1-\ell}}} K^{\frac{r}{r+1-\ell}} V_{I}^{\frac{1-\ell}{r+1-\ell}} & \text { if } m(I) \geq 2\left[\frac{(1-\ell) K}{r V_{I}}\right]^{\frac{1}{r+1-\ell}}, \\ \frac{2^{1-\ell} K}{[m(I)]^{1-\ell}}+\frac{V_{I}[m(I)]^{r}}{2^{r}} & \text { if } 0<m(I)<2\left[\frac{(1-\ell) K}{r V_{I}}\right]^{\frac{1}{r+1-\ell}} .\end{cases}
$$


Proof. We know, from the proof of Theorem 2, that

$$
\left|f^{\prime}(a)\right| \leq \frac{|f(b)-f(a)|}{|b-a|}+V_{I}|b-a|^{r}, \quad \text { for all } a, b \in I, a \neq b .
$$

Using the hypothesis (4.1), we conclude that

$$
\left|f^{\prime}(a)\right| \leq \frac{K}{|b-a|^{1-\ell}}+V_{I}|b-a|^{r}
$$

for any $a, b \in I, a \neq b$.

By a similar argument to the one used in proving Theorem 2, we conclude that

$$
\left|f^{\prime}(a)\right| \leq \inf _{s \in\left(0, \frac{m(I)}{2}\right)}\left[\frac{K}{s^{1-\ell}}+V_{I} s^{r}\right]=M
$$

for any $a \in I$.

If we now apply Lemma 1 for $C=K, u=1-\ell, D=V_{I}$, we observe that

$$
\begin{aligned}
& \inf _{s \in\left(0, \frac{m(I)}{2}\right)}\left[\frac{K}{s^{1-\ell}}+V_{I} s^{r}\right] \\
& = \begin{cases}\frac{r+1-\ell}{(1-\ell)^{\frac{1-\ell}{r+1-\ell}} r^{\frac{r}{r+1-\ell}}} K^{\frac{r}{r+1-\ell}} V_{I}^{\frac{1-\ell}{r+1-\ell}} \text { if } \frac{m(I)}{2} \geq\left(\frac{(1-\ell) K}{r V_{I}}\right)^{\frac{1}{r+1-\ell}}, \\
\frac{K}{\left(\frac{m(I)}{2}\right)^{1-\ell}}+V_{I}\left(\frac{m(I)}{2}\right)^{r} & \text { if } \frac{m(I)}{2}<\left(\frac{(1-\ell) K}{r V_{I}}\right)^{\frac{1}{r+1-\ell}} .\end{cases}
\end{aligned}
$$

and the inequality (4.2) is obtained.

The following corollary holds.

Corollary 3. Let $I$ be an interval in $\mathbb{R}$ and $f: I \rightarrow \mathbb{R}$ be a locally absolutely continuous function on I. If $f^{\prime} \in L_{p}(I), p>1$ and if $f^{\prime}$ is of locally bounded variation and the condition (3.1) holds, then $f^{\prime} \in L_{\infty}(I)$ and

$$
\left\|f^{\prime}\right\|_{I, \infty} \quad \leq\left\{\begin{array}{l}
\frac{p r+1}{p^{\frac{p r}{p r+1}} r^{\frac{p r}{p r+1}}}\|f\|_{I, p}^{\frac{p r}{p r+1}} V_{I}^{\frac{1}{p r+1}} \text { if } m(I) \geq 2\left(\frac{\|f\|_{I, p}}{p r V_{I}}\right)^{\frac{p}{p r+1}}, \\
\frac{2^{\frac{1}{p}}\|f\|_{I, p}}{[m(I)]^{1-\ell}}+\frac{V_{I}[m(I)]^{r}}{2^{r}} \quad \text { if } 0<m(I)<2\left(\frac{\|f\|_{I, p}}{p r V_{I}}\right)^{\frac{p}{p r+1}} .
\end{array}\right.
$$


Proof. If $f^{\prime} \in L_{p}(I)$, then we have

$$
\begin{aligned}
|f(b)-f(a)| & =\left|\int_{a}^{b} f^{\prime}(s) d s\right| \leq\left|\int_{a}^{b}\right| f^{\prime}(s)|d s| \\
& \leq\left.\left.|b-a|^{\frac{1}{q}}\left|\int_{a}^{b}\right| f^{\prime}(s)\right|^{p} d s\right|^{\frac{1}{p}} \\
& \leq|b-a|^{1-\frac{1}{p}}\left\|f^{\prime}\right\|_{I, p}, \quad p>1, \frac{1}{p}+\frac{1}{q}=1,
\end{aligned}
$$

for a.e. $a, b \in I$.

Using Theorem 3 for $\ell=1-\frac{1}{p}$ and $K=\left\|f^{\prime}\right\|_{I, p}$, we deduce the desired result (4.6).

The following result may be proved as well.

Corollary 4. With the assumptions in Corollary 3 , and if $f^{\prime} \in L_{1}(I)$, then $f^{\prime} \in$ $L_{\infty}(I)$ and

$$
\left\|f^{\prime}\right\|_{I, \infty} \leq\left\{\begin{array}{l}
\frac{r+1}{r^{\frac{r}{r+1}}}\left\|f^{\prime}\right\|_{I, 1}^{\frac{r}{r+1}} V_{I}^{\frac{1}{r+1}} \quad \text { if } \quad m(I) \geq 2\left(\frac{\left\|f^{\prime}\right\|_{I, 1}}{r V_{I}}\right)^{\frac{1}{r+1}}, \\
\frac{2\left\|f^{\prime}\right\|_{I, 1}}{m(I)}+\frac{V_{I}[m(I)]^{r}}{2^{r}} \text { if } \quad 0<m(I)<2\left(\frac{\left\|f^{\prime}\right\|_{I, 1}}{r V_{I}}\right)^{\frac{1}{r+1}} .
\end{array}\right.
$$

\section{References}

[1] Z. Ditzian, Remarks, questions and conjections on Landau-Kolmogorov-type inequalities, Math. Ineq. Appl. 3(2000), 15-24.

[2] S. S. Dragomir, The Ostrowski integral inequality for mappings of bounded variation, Bull. Austral. Math. Soc. 60(1999), 145-156.

[3] S. S. Dragomir, On the Ostrowski's integral inequality for mappings with bounded variation and applications, Math. Ineq. \& Appl. 4(2001), 59-66.

[4] S. S. Dragomir and C. I. Preda, Some Landau type inequalities for functions whose derivatives are Hölder continuous, Non. Anal. Forum (Korea) 9(2004), 25-31.

[5] G. H. Hardy and J. E. Littlewood, Some integral inequalities connected with the calculus of variations, Quart. J. Math. Oxford Ser. 3(1932), 241-252.

[6] G. H. Hardy, E. Landau and J. E. Littlewood, Some inequalities satisfied by the integrals or derivatives of real or analytic functions, Math. Z. 39(1935), 677-695.

[7] R. R. Kallman and G.-C. Rota, On the inequality $\left\|f^{\prime}\right\|^{2} \leq 4\|f\| \cdot\left\|f^{\prime \prime}\right\|$, in "Inequalities", vol. II (O. Shisha, Ed), 187-192. Academic Press, New York, 1970.

[8] E. Landau, Einige Ungleichungen für zweimal differentzierban funktionen, Proc. London Math. Soc. 13(1913), 43-49.

[9] C. P. Niculescu and C. Buşe, The Hardy-Landau-Littlewood inequalities with less smoothness, J. Inequal. in Pure and Appl. Math. 4(2003), No. 3, Article 51, [ONLINE: http://jipam.vu.edu.au/article.php?sid=289]. 
[10] D.S. Mitrinović, J. E. Pečarić and A. M. Fink, Inequalities Involving Functions and their Integrals and Derivatives, Kluwer Academic Publishers, Dordrecht/Boston/London, 1991.

School of Computer Science and Mathematics, Victoria University, PO Box 14428, Melbourne VIC 8001, Australia.

E-mail: neil.barnett@vu.edu.au

School of Computer Science and Mathematics, Victoria University, PO Box 14428, Melbourne VIC 8001, Australia.

E-mail: sever.dragomir@vu.edu.au 